$p<0.001$ ), moderate correlations were found with HADS-A and HADS-D (HADS-A $(r=0.618, p<0.001)$, HADS-D $(r=0.507, p<0.001))$. Low correlation was found between BETY-BQ and HAQ $(r=286, p=0.003)$ (Table 2).

Table1. Descriptive Statistics

\begin{tabular}{lcccc}
\hline & Minimum & Maximum & Mean & Standart Deviation \\
\hline Age (years) & 20 & 60 & 44.98 & 11.04 \\
Height $(\mathrm{cm})$ & 145.00 & 190.00 & 165.61 & 8.88 \\
Weight $(\mathrm{kg})$ & 44 & 138 & 75.54 & 17.36 \\
VKI $\left(\mathrm{kg} / \mathrm{m}^{2}\right)$ & 17.85 & 49.00 & 27.54 & 6.02 \\
\hline
\end{tabular}

Table 2.

\begin{tabular}{lcccc}
\hline & PSAQOL & HADS-A & HADS-D & HAQ \\
\hline BETY-BQ & $r 0.826^{\star *}$ & $r=0.618^{\star *}$ & $r=0.507^{\star *}$ & $r=286^{\star *}$ \\
& $p<0.001$ & $p<0.001$ & $p<0.001$ & $p=0.003$
\end{tabular}

${ }^{* *}$ Correlation is significant at the 0.01 level (2-tailed)

Conclusion: In this study, it was found that BETY-BQ is a scale that can be used in total to evaluate the quality of life, emotion-status level and functionality of individuals diagnosed with PSA. It was thought that BETY-BQ could be considered and preferred by clinicians when individuals with PsA should be evaluated from a biopsychosocial point of view.

References:

[1] Unal E, Arın G, Karaca Nb, Kiraz S, Akdogan A, Kalyoncu U, et al. Development of a quality of life measurement for rheumatic patients: item pool construction Journal of Exercise Therapy and Rehabilitation. 2017; 4 (2): 67-75.

Disclosure of Interests: Senem Bulut: None declared, Edibe Ünal: None declared, Aykut Özçadırcı: None declared, Umut Kalyoncu Consultant of: Abbvie, Amgen, Janssen, Lilly, Novartis, UCB

DOI: 10.1136/annrheumdis-2020-eular.5609

\section{FRI0620-HPR THE EFFECT OF THERMOFORMABLE FOOT ORTHOSES ON WALKING IN RHEUMATOID ARTHRITIS PATIENTS: PRELIMINARY RESULTS FROM AN OPEN CLINICAL TRIAL}

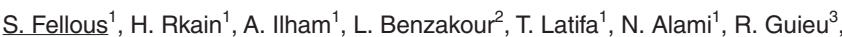
Y. Jammes ${ }^{3}$, N. Hajjaj-Hassouni ${ }^{4}$, F. Allali ${ }^{1} .{ }^{1}$ Faculty of Medicine and Pharmacy of Rabat, Mohammed V University, Rabat, Department of Rheumatology B, EI Ayachi Hospital, Rabat, Morocco; ${ }^{2}$ PROTEOR Morocco, Casablanca, Morocco; ${ }^{3}$ University Aix-Marseille, Marseille, UMR MD2 Faculty of Medecine,, Marseille, France; ${ }^{4}$ International university of Rabat, Rabat, Morocco

Background: Foot pain is common in rheumatoid arthritis and appears to persist despite modern day medical management.

Objectives: To evaluate the impact of thermoformable foot orthoses on walking in rheumatoid arthritis (RA) patients.

Methods: This is a open clinical trial, that included 14 consecutive patients $(85,7 \%$ female, mean age 54,8 \pm 10 years) with RA (median duration of progression of 9 [5-12] years), the average DAS28 was $2,7+/-1,2$ and the functional impact objectified by the Health Assessment Questionnaire $(\mathrm{HAQ})$ was on average $0.9 \pm 0.7$. The foot problem was bilateral in $100 \%$ and inaugural in $85.7 \%$ of the cases. The 14 rheumatoid subjects were examined and appropriate foot orthoses were prescribed according to each patient's needs. All the patients were evaluated at baseline and 8 weeks after use of orthoses. Gait pain, difficulty walking and the 10 Meter Walk test were noted at each appointment.

We used dynamic baropodometric analysis to assess postural evaluation. We calculated the lateral-medial index of each foot before and after the use of orthoses.

Table 1. Assessment of walking before and after the use of orthoses

\begin{tabular}{lccc}
\hline & Before orthoses & After orthoses & $p$ \\
\hline Pain when walking*(EVA 0-10) & $5[3-5,2]$ & $0[0-2]$ & $\mathbf{0 , 0 0 2}$ \\
Difficulty walking ${ }^{*}(0-10)$ & $4[3-5,2]$ & $2[0-2]$ & $\mathbf{0 , 0 0 2}$ \\
- In house & $6[4,7-7]$ & $2[1,5-2,5]$ & $\mathbf{0 , 0 2}$ \\
- Outside & & & \\
10 Meter Walk test**(Normal comfortable speed) & $18,64 \pm 3,7$ & $16,9 \pm 5$ & 0,2 \\
- Nomber of steps & $11,9 \pm 4,6$ & $11,8 \pm 5,2$ & 0,9 \\
- Duration (sec) & $56,4 \pm 17,7$ & $58,6 \pm 20,3$ & 0,6 \\
- Walking speed (m/min) & & & \\
latero-medial (L/M) index & $1,18 \pm 0,17$ & $1,23 \pm 0,23$ & 0,1 \\
- L/M index of the right foot & $1,25 \pm 0,17$ & $1,26 \pm 0,19$ & 0,9 \\
- L/M index of the left foot & & &
\end{tabular}

*median and quartile

**average and standard deviation

p significant if $<0,05$
Results: A significant decrease in walking pain $(p=0.002)$ and difficulty walking $(p=0.02)$ was found with the use of orthoses. The variations in 10 meter walk test and dynamic baropodometric parameters were not significant $(p>0,05)$.

There were no significant correlations between pain and difficulty walking, the progression of RA, the duration of foot damage and the functional impact meas ured by the HAQ.

Conclusion: Thermoformable foot orthoses significantly reduced pain and difficulty walking. The absence of factors associated with pain and difficulty walking could possibly be related to the small sample size.

Disclosure of Interests: None declared

DOI: 10.1136/annrheumdis-2020-eular.5698

\section{FRI0621-HPR CHANGES IN LOCUS OF CONTROL LEVEL IN PATIENTS WITH RHEUMATOID ARTHRITIS AFTER THE COURSE OF BIOFEEDBACK TRAINING}

R. Grekhoff'. 'Zborovsky' Research Institute for Clinical and Experimental Rheumatology, Volgograd, Russian Federation

\section{Background:}

Objectives: Our aim was to study the effect of biofeedback (BFB) training on the locus of control in patients suffering from rheumatoid arthritis (RA), and to justify the use of this method in the complex treatment of the disease.

Methods: 40 RA patients hospitalized in the rheumatology department were examined. The average age of patients was 48.6 years \pm 7.73 years (from 30 to 70 years), women accounted for the majority $=26(86 \%)$, the average duration of the disease was 12 years \pm 3.44 years. We use J. Rotter's Locus of Control Scale in E.F.Bazhin adaptation. RA patients were divided into two groups: the main (20 patients) and control (20 patients). Patients of the main group received complex therapy with 12 sessions of BFB training, mainly based on the parameters of the brain's electrical activity - EEG relaxation using the Reakor ${ }^{\mathrm{TM}}$ psychophysiological rehabilitation complex manufactured by Medicom MTD (Taganrog).

Results: We revealed externality in RA patients in the general field $(3.03 \pm 0.3)$ as well as in the field of relation to the disease $(3.86 \pm 0.23)$ and in the field of production relations $(3.43 \pm 0,25)$. After BFB trainings, an increase in internality was observed on the scales of the general sphere $(p<0.05)$ and attitude to the disease $(p<0.01)$ in patients of the main group. In the group of patients receiving conventional treatment, the dynamics of the results was unreliable.

Conclusion: It should be noted that the locus of control (or subjective control) is a quality that characterizes a person's tendency to attribute responsibility for the results of his activity to external forces, or to his own abilities and efforts. Externality is manifested when people prefer to shift responsibility for important events of their life to external circumstances, and external forces (bosses, colleagues, etc.). In the field of attitude to the disease, externality is manifested when patien behaves passively, and believes that he cannot influence the course of the disease in any way, shifting all responsibility for the treatment results to medical staff, which can lead to non-compliance with the treatment regimen and an increase in the level of anxiety and depression, decreased self-esteem. The onset of the disease and its associated social consequences (disability, loss of social roles, etc.) can cause a negative mental state of learned helplessness. Learned helplessness is defined as a condition that occurs as a result of uncontrolled, mainly negative events, which manifests itself in violations of emotional, motivational and cognitive processes. In other words, RA patient suffering from this condition expects treatment failures and reduces control over compliance with the treatment regimen. BFB therapy can be used in order to correct and prevent the state of learned helplessness by increasing the level of internality.

It is assumed that increasing internality in the BFB process is associated with teaching the patient the skills of self-regulation of physiological processes. The mechanisms of BFB therapeutic effect are not only changes in physiologica parameters (improvement of cerebral and peripheral blood flow, muscle relaxation, and improvement of sleep) but also in a shift in the locus of control from external to internal, which can increase compliance, reduce neurotic complaints, mobilize volitional potential and improve patient self-esteem.

As a result of BFB course, an increase in the internality was noted in patients on the scales of the general sphere and the sphere of attitude to the disease. It is advisable to use the BFB to increase the compliance and effectiveness of complex treatment of RA patients.

Disclosure of Interests: None declared

DOI: 10.1136/annrheumdis-2020-eular.1571

\section{FRI0622-HPR IMPACT OF A PHARMACIST'S INTERVENTION ON THE KNOWLEDGE OF BIOLOGICS AND ADHERENCE IN PATIENTS WITH SPONDYLOARTHRITIS: A} RANDOMIZED, OPEN-LABEL, CONTROLLED TRIAL

L. Gutermann ${ }^{1}$, S. Dumas ${ }^{2}$, C. López-Medina ${ }^{3}$, L. Boissinot ${ }^{4}$, C. Cotteret ${ }^{5}$, V. Perut ${ }^{6}$, A. Moltó ${ }^{3}$, O. Conort ${ }^{2}$, M. Dougados ${ }^{3} .{ }^{1}$ Hôpital Marie Lannelongue, Pharmacy, Le Plessis Robinson, France ${ }^{2}$ Hôpital Cochin - APHP, Pharmacy, 
Paris, France; ${ }^{3}$ Hôpital Cochin - APHP, Rheumatology, Paris, France; ${ }^{4}$ Hôpital Sud-Francillien, Pharmacy, Corbeil-Essonnes, France; ${ }^{5}$ Hôpital Necker - Enfants Malades, Pharmacy, Paris, France; ${ }^{6}$ Hôpital Cochin - APHP, Risk and quality management, Paris, France

Background: In chronic rheumatic diseases, non-adherence to treatment is associated with a progression of disease and an increased morbidity (1). In spondyloarthritis (SpA), improving patients' knowledge on their subcutaneous biologic disease-modifying antirheumatic drugs (bDMARDs) is a key factor to enhance medication adherence (2). The patient information has to ensure the acquisition of safety skills regarding their treatment management.

Objectives: To evaluate the impact of a pharmacist's educational interview on knowledge and therapeutic adherence of subcutaneous bDMARDs in patients with SpA.

Methods: Population and study design: consecutive adult patients with well-controlled axial SpA, stable on subcutaneous bDMARDs were enrolled in a randomized, controlled, single-center, open-label, 6-months trial. Intervention: A pharmacist's educational interview provided information on bDMARDs management at baseline in the intervention group (IG) and at month 6 (M6) in the control group (CG). A booklet containing essential information was given to the patient. Intervention allocation: After written consent, the study treatment was randomly allocated via a computer program by simple randomization, with an allocation ratio of 1:1. Outcome measures: The change of a weighted knowledge score $(0$ -100) concerning the bDMARDs management and the change in the Medication Possession Ratio (MPR) at M6 were primary outcomes. The changes in disease activity (BASDAI) and patients' satisfaction regarding the pharmacists' interview were secondary outcomes. Statistics: Changes in knowledge score, MPR and BASDAI were compared between the two groups using the T-Student test. Statistical analysis was performed in intention-to-treat. Missing data was handled with multiple imputations.

Results: Patients' characteristics at baseline were comparable among the 89 included patients (46 in IG, 43 in CG). The means \pm SD of the knowledge score were $75.3 \pm 14.2$ versus $73.0 \pm 13.2$ and $86.3 \pm 12.6$ versus $76.0 \pm 14.1$ in the IG versus $C G$ at baseline and at M6, respectively. The patient's knowledge score improved at a greater magnitude in the IG $(+11.0 \pm 11.5$ versus $+3.0 \pm 10.6$ in the $I G$ versus the $C G$ respectively, $p<0.0001)$. The MPR at baseline were very high in both groups $(92.9 \pm 14.6 \%$ versus $96.6 \pm 15.6 \%$ in the $I G$ versus the $C G$, respectively). There was a trend in a better adherence $(+2.2 \pm 13.9$ versus $-0.6 \pm 18.9$ in the IG versus the CG in the MPR score respectively, $p=0,691$ ). The disease activity (changes in BASDAI) remained stable during the study in both groups. All the patients were mostly or totally satisfied by the pharmacists' interview.

Conclusion: Pharmacists' educational interview on subcutaneous bDMARDs is effective in improving the knowledge of patients with SpA on their treatment. Regarding therapeutic adherence, a trend in favor of an improvement was observed in the intervention group but did not reach the statistically significance. Nevertheless, the results observed in this study are an argument to propose to include the pharmacists in the multidisciplinary team in charge of the management of patients with SpA.

References:

[1] Bluett J, Morgan C, Thurston L et al. Impact of inadequate adherence on response to subcutaneously administered anti-tumour necrosis factor drugs: results from the biologics in rheumatoid. Rheumatology. 2015;54(3):494-9.

[2] Gossec L, Molto A, Romand X et al. Recommendations for the assessment and optimization of adherence to disease-modifying drugs in chronic inflammatory rheumatic diseases: A process based on literature reviews and expert consensus. Joint Bone Spine. 2019;86(1):13-9.

Disclosure of Interests: Loriane Gutermann: None declared, Sophie Dumas: None declared, Clementina López-Medina: None declared, Léa Boissinot: None declared, Camille Cotteret: None declared, Valérie Perut: None declared, Anna Moltó Grant/research support from: Pfizer, UCB, Consultant of: Abbvie, BMS, MSD, Novartis, Pfizer, UCB, Ornella Conort: None declared, Maxime Dougados Grant/research support from: AbbVie, Eli Lilly, Merck, Novartis, Pfizer and UCB Pharma, Consultant of: AbbVie, Eli Lilly, Merck, Novartis, Pfizer and UCB Pharma, Speakers bureau: AbbVie, Eli Lilly, Merck, Novartis, Pfizer and UCB Pharma DOI: 10.1136/annrheumdis-2020-eular.430

\section{FRI0623-HPR HEALTHCARE RESOURCE USE IN PATIENTS WITH TRAPEZIOMETACARPAL OSTEOARTHRITIS}

T. Hamasaki ${ }^{1,2,3}$, P. Harris ${ }^{1,2,4}$, N. Bureau ${ }^{1,2,3}$, N. Gaudreault ${ }^{5,6}$, N. Patenaude ${ }^{5,6,7}$, M. Choinière ${ }^{2,3} \cdot{ }^{1}$ CHUM Hospital (Université de Montréal), Montréal, Canada; ${ }^{2}$ Université de Montréal, Montréal, Canada; ${ }^{3}$ Centre de recherche du CHUM (CRCHUM), Montreal, Canada; ${ }^{4}$ Research Centre of the CHUM, Montreal, Canada; ${ }^{5}$ Université de Sherbrooke, Sherbrooke, Canada; ${ }^{6}$ Centre de recherche du CHUS (CRCHUS), Sherbrooke, Canada; ${ }^{7} \mathrm{CHUS} \mathrm{Hospital} \mathrm{(Université} \mathrm{de}$ Sherbrooke), Sherbrooke, Canada
Background: Trapeziometacarpal osteoarthritis (TMO) is one of the most debilitating forms of hand osteoarthritis (OA). According to the recent EULAR guidelines ${ }^{1,2}$ and a systematic review, ${ }^{3}$ the efficacy of topical/oral non-steroid anti-inflammatory drugs (NSAIDs), orthoses, hand exercises, and psychosocial interventions for hand OA or TMO are supported by scientific evidence. Cortisone injections and acetaminophen are generally not recommended. ${ }^{1{ }^{3}}$ Besides, TMO management is suboptimal: only $21 \%$ of patients receive rehabilitative interventions prior to referral to hand surgeons. ${ }^{4}$

Objectives: We aimed at documenting the types of treatment TMO patients employ and their healthcare resource use.

Methods: A total of $227 \mathrm{TMO}$ patients recruited from 16 healthcare institutions completed a questionnaire about 1) received interventions, 2) analgesic strategies, and 3) healthcare professional consultations.

Results: Acetaminophen (64.3\% of the participants), oral NSAIDs (31.7\%), topical NSAIDs $(11.9 \%)$, and nutraceuticals $(7.9 \%)$ were the most commonly used medications. More than $70 \%$ of the participants reported having received cortisone injection(s) $(72.5 \%)$ and orthosis $(75.7 \%)$. More than half employed hand exercises massage and heat/cold application. Relaxation/respiration, meditation, distraction, assistive devices, and joint protection principles were used by smaller percentages of participants (13.0-30.9\%). Patients with TMO reported having consulted various types of healthcare professionals: family physicians, plastic/orthopaedic surgeons, radiologists-interventionists, rheumatologists, physiatrists, occupational/physical therapists, osteopaths, chiropractors, pharmacists, and acupuncturists. Only $4.8 \%$ of the participants reported having received psychosocial interventions.

Conclusion: TMO patients use numerous types of modalities to relieve their pain. Provision of evidence-based interventions tailored to their needs is clearly needed.

References:

[1] Kloppenburg M, Kroon FP, Blanco FJ, et al. 2018 update of the EULAR recommendations for the management of hand osteoarthritis. Annals of the rheumatic diseases. 2019;78:16-24.

[2] Geenen R, Overman CL, Christensen R, et al. EULAR recommendations for the health professional's approach to pain management in inflammatory arthritis and osteoarthritis. Annals of the rheumatic diseases. 2018;77:797-807.

[3] Hamasaki T, Laprise S, Harris PG, et al. Efficacy of non-surgical interventions for trapeziometacarpal (thumb base) osteoarthritis: A systematic review. Arthritis Care \& Research. 2019; In press (https://doi.org/10.1002/acr.24084).

[4] Gravås EMH, Tveter AT, Nossum R, et al. Non-pharmacological treatment gap preceding surgical consultation in thumb carpometacarpal osteoarthritis - a cross-sectional study. BMC musculoskeletal disorders. 2019;20:180-180. Acknowledgments: This study was supported by a discretionary fund of the Centre de recherche du CHUM (CRCHUM) to Choinière and from the Multidisciplinary Council of the CHUM. Hamasaki was supported by a Doctoral training award of the Fonds de recherche du Québec-Santé, a doctoral scholarship from the CHUM Foundation to Harris (Hand Surgery Branch) and from Choinière's internal funds of the CRCHUM.

Disclosure of Interests: None declared

DOI: 10.1136/annrheumdis-2020-eular.556

\section{FRI0624-HPR A SYSTEMATIC REVIEW OF JOB LOSS PREVENTION INTERVENTIONS FOR PERSONS WITH INFLAMMATORY ARTHRITIS.}

C. M. T. Madsen ${ }^{1,2}$, S. Kjaer ${ }^{3}$, J. Primdahl1,2,4 J. R. Christensen ${ }^{5}$, C. Von Bülow ${ }^{3,6}$ ${ }^{1}$ Danish Hospital for Rheumatic Diseases, Sønderborg, Denmark; ${ }^{2}$ Department of Regional Health Research, University of Southern Denmark, Odense, Denmark; ${ }^{3}$ Research Initiative for Activity Studies and Occupational Therapy, Research unit for General Practice, Department of Public Health, University of Southern Denmark, Odense, Denmark; ${ }^{4}$ Hospital of Southern Jutland, University Hospital of Southern Denmark, Aabenraa, Denmark; ${ }^{5}$ Research unit for General Practice, Department of Public Health, University of Southern Denmark, Odense, Denmark; ${ }^{6}$ The Parker Institute, Copenhagen University Hospital, Bispebjerg and Frederiksberg, Denmark

Background: Persons with inflammatory arthritis (IA) have a higher level of absenteeism from work than those without IA and up to 20-30\% become permanently work-disabled during the first years after being diagnosed with IA. Despite developments of new pharmacological and surgical treatments, people with IA still report reduced work ability. It is therefore relevant to offer effective interventions designed to prevent job loss and improve work function (i.e. job loss prevention interventions) to support people with IA to stay connected to the labour market. Initial effects of job loss prevention interventions have been established in a Cochrane review by Hoving et al. 2014 (1), but as only three randomized controlled trials (RCT) were identified, it seems relevant to investigate if new evidence has emerged. 